\title{
La importancia y el destino de los resúmenes presentados en reuniones científicas
}

\author{
HUMBERTO REYES B. ${ }^{1}$, MAX ANDRESEN H. ${ }^{2}$, JOAQUÍN PALMA H. ${ }^{2}$ \\ The relevance and fate of abstracts presented \\ in scientific meetings
}

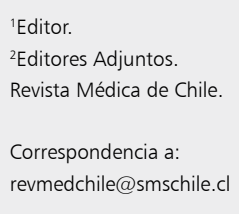

Abstracts presented in scientific meetings are indispensable tools to diffuse the latest research in the field. They provide the authors with an opportunity to receive feedback from a critical audience so they can prepare a final manuscript to be submitted to a peer-reviewed journal. However, several studies in a wide range of medical specialties and other related sciences showed that no more than $50 \%$ of abstracts presented in annual meetings of learned societies are published in a 5-year follow up after the meeting. Therefore, abstracts are considered "preliminary publications" and it is recommended not to include them as bibliographic references unless they have been published recently (less than 3 years) in peer-reviewed journals (regular issues or supplements) or in their official websites. Databases dependent of the National Library of Medicine (USA) or SciELO do not index individual abstracts from a meeting. Authors and reviewers should be reminded that manuscripts that have shaped current knowledge probably had also been presented as abstracts in scientific meetings, sometime before their final publication.

(Rev Med Chile 2011; 139: 7-10).

Key words: Bibliography; Manuscripts; Meeting abstracts; Periodicals.

L os resúmenes de trabajos presentados en una reunión científica (congreso, simposio, o seminario) tienen una importancia crucial para la reunión y para sus autores.

Los organizadores y los asistentes a la reunión tienen una oportunidad de conocer la producción científica reciente en su ámbito profesional; identificar a quienes participan en dicha producción, particularmente a los autores noveles que se incorporan a la sociedad respectiva; registrar sus intereses de trabajo y así poder seleccionar los temas a tratar en futuras reuniones. Los autores pretenden comunicar una novedad que observaron, una tecnología nueva que aplicaron, o la experiencia que reunieron en una materia. Por ende, la variedad del contenido y estructura de los resúmenes puede abarcar desde la presentación de un caso clínico hasta la de un trabajo con casuística numerosa o con observaciones en modelos experimentales.

Para los autores, estos resúmenes les permiten exponer lo expresado en el párrafo previo ante una audiencia potencialmente crítica, siendo oportunidades educacionales para estudiantes de medicina y médicos que se inician en investigación y otras actividades académicas. Además, los resúmenes sustentarán su posibilidad de asistir al evento (particularmente importante cuando ocurre en una localidad alejada de su lugar de residencia), incluyendo la ayuda financiera para ello. Quienes participan en una carrera académica necesitan un respaldo institucional, administrativo y financiero, para asistir a reuniones científicas; dicho respaldo generalmente exige ser autor de un trabajo que se presentará en la reunión respectiva. Otro posible logro para los autores está en que las reuniones societarias suelen otorgar premios a presentaciones 
elegidas por comisiones ad hoc, lo que citarán en sus antecedentes curriculares.

La suma de ambos intereses da vida a los congresos y otras reuniones científicas, les provee de público interesado por asistir a presentaciones orales y visitar carteles ("posters"). En el balance post reunión, el número de "presentaciones libres" que atrajo al programa suele emplearse como uno de los criterios para evaluar su éxito. Sin estas presentaciones, las reuniones científicas se convierten en una sucesión de conferencias, mesas redondas y otros tipos de discusiones grupales insuficientes para mantener el interés de los asistentes, que no se sienten "tan" participantes como cuando tienen una presentación propia.

\section{¿Cuánto tiempo sobrevive una presentación después de una reunión científica?}

Desde el siglo 17 se sabe que un trabajo presentado en una reunión científica debe quedar registrado por escrito, o su existencia no será reconocida. De allí que es fundamental su difusión impresa o en una página electrónica estable. Las formas tradicionales de registrar las presentaciones en reuniones científicas son su publicación en un "libro de resúmenes" o en una revista, sea en un número regular o en un suplemento.

Una vez que un resumen queda así registrado, sus autores esperan que otros autores interesados en el tema lo incluyan entre las futuras referencias bibliográficas y, por supuesto, desean citarlo en sus antecedentes curriculares y en sus propias publicaciones. Para que el resumen sea aceptable como referencia bibliográfica por las revistas médicas adscritas a los "Uniform Requirements for Manuscripts Submitted to Biomedical Journals" (URM) establecidos por el International Committee of Medical Journal Editors, debe ser publicado en una revista que aplica la revisión por pares o en un sitio electrónico equivalente. Los "libros de resúmenes" no cumplen este requisito, porque no son publicaciones con accesibilidad permanente y no se registran en bibliotecas o bases de datos.

Si el resumen se publica en una revista indexada en bases de datos, sus autores esperan que sea reproducido por las bases de datos, tal como los manuscritos finales publicados en esa revista. Pero las bases de datos con relevancia internacional no reproducen estos resúmenes ni enlistan sus autores y su título. Vale la pena dejarlo en claro, principalmente para los autores de resúmenes presentados en congresos y otras reuniones científicas que se publiquen en la Revista Médica de Chile: no son indexados por la National Library of Medicine (MEDLINE) ni por SciELO Chile. Ello implica que están accesibles para los lectores de la versión impresa de la Revista, pero no para quienes hagan una búsqueda bibliográfica en PubMed ni en la página web www.scielo.cl. Para los resúmenes presentados en las reuniones anuales (o con otra periodicidad regular) de sociedades científicas y que ocupan un número completo de su revista (o un suplemento específico para dicha reunión), la National Library of Medicine crea una cita especial que indica la numeración de páginas que incluyen a todos los resúmenes publicados, tal como lo ilustra este ejemplo:

Hepatology. 2010 Oct;52 Suppl:320A-1291A Abstracts of the American Association for the Study of Liver Diseases 61st Annual Meeting and Postgraduate Course. October 29-November 2, 2010. Boston, Massachusetts, USA.

[No authors listed]

PMID: 20949695 [PubMed - indexed for MEDLINE] Publication Types:

- Congresses

- Overall

\section{¿Por qué las bases de datos no indexan estos resúmenes individualmente?}

La razón principal es que no cumplen los requisitos de ser publicaciones finales, con datos completos, que fueron revisados por pares, sus críticas fueron dadas a conocer a los autores quienes re-enviaron un manuscrito corregido y los editores de la revista respectiva aceptaron su publicación. Los resúmenes presentados en reuniones científicas se consideran publicaciones preliminares cuya evaluación no tuvo la estrictez que se aplica a los manuscritos definitivos: cuando un experto revisa un manuscrito final, que ha sido enviado a publicación en una revista, suele ser más meticuloso y crítico que cuando se le pide seleccionar resúmenes para ser presentados en una reunión científica.

En distintas especialidades de la medicina (por ej: Anestesia, Cardiología, Cirugía, Farmacología, Medicina Intensiva, Neumología, Neurología y 
Neurocirugía, Oftalmología, Oncología, Ortopedia, Pediatría) y en otras profesiones (por ej: Medicina Veterinaria, Química Clínica) se ha estudiado el destino que tuvieron los resúmenes presentados en sus reuniones científicas, para verificar si dieron o no origen a una publicación in extenso y en cuanto tiempo ${ }^{1-13}$. Sólo 30 a $50 \%$ de los resúmenes originaron una publicación in extenso, buscada hasta 5 años después de la reunión respectiva. Ese lapso daría el tiempo suficiente para que un estudio preliminar se complete y su manuscrito final cumpla el proceso de revisión editorial en una revista, incluso en una segunda revista si no fue aceptado por la primera. Para los resúmenes que originaron publicaciones in extenso, el intervalo entre su presentación inicial y la publicación final tuvo una mediana de 14 a 16 meses. Los ensayos clínicos con personas asignadas al azar ("aleatorizados") o controlados tuvieron una tasa de publicación mayor (63\%) que el total de los resúmenes ${ }^{6,8}$.

Algunos trabajos preguntaron a los autores de resúmenes no publicados in extenso su explicación para ello ${ }^{4,9,11}$. La razón más frecuente fue falta de tiempo, de financiamiento o de otros recursos (casi $40 \%$ ), seguida por una falta de interés (alrededor de 34\%). Respuestas menos frecuentes fueron "el estudio aún no ha conseguido un número adecuado de observaciones"; "en el congreso nos hicieron críticas que nos desalentaron”. Llama la atención que sólo $4 \%$ adujeron que hubo un manuscrito final pero fue rechazado por una revista médica y los autores no insistieron en otra.

Con estos antecedentes, es obvio que no hay expertos que puedan pronosticar el destino final de los resúmenes presentados en una reunión científica y tampoco pueden las bases de datos seleccionar los resúmenes según su potencialidad de generar un manuscrito definitivo.

\section{${ }_{¿}$ Pueden citarse como referencias bibliográficas los resúmenes presentados en reuniones científicas?}

La respuesta es afirmativa, pero con salvedades y advertencias. Los "URM" recomiendan "Avoid using abstracts as references", pero no los proscriben. Un resumen que fue publicado en una revista con acceso universal, que contiene información original, novedosa y oportuna, y que es importante para un manuscrito, puede ser incluido entre sus referencias bibliográficas si fue publicado recientemente (tal vez dentro de los últimos 3 años). En este plazo el manuscrito final puede hallarse en elaboración o estar siendo revisado en alguna revista. Pero cuando un resumen tiene una antigüedad mayor a 3 años debemos pensar que si no dio origen a una publicación in extenso... por algo será.

Debemos recordar que aquellas publicaciones que han tenido una trascendencia enorme para el progreso de la medicina o de las ciencias, alguna vez fueron resúmenes presentados en reuniones científicas (todo cisne fue polluelo). Por ende, al elegir las referencias para nuestras propias publicaciones debemos juzgar la relevancia de los resúmenes tal como juzgamos a los manuscritos in extenso, recordando que "la presentación de las referencias... exige un esfuerzo de selección, dando preferencia a la originalidad y trascendencia de las publicaciones citadas y a su relación estricta con el aspecto del tema que se está abordando" ${ }^{11}$.

\section{Referencias}

1. Goldman L, Loscalzo A. Fate of Cardiology research originally published in abstract form. N Engl J Med 1980; 303(5): 255-9.

2. Weintraub WH. Are published manuscripts representative of the surgical meeting abstracts? An objective appraisal. J Pediatr Surg 1987; 22: 11-13.

3. Scherer RW, Dickersin K, Langenberg P. Full publication of results initially presented in abstracts: A meta-analysis. JAMA 1994; 272(2): 158-62.

4. Weber EJ, Callaham ML, Wears RL, Barton C, Young G. Unpublished research from a medical specialty meeting. Why investigators fail to publish. JAMA 1998; 280: 257-9.

5. Bhandari M, Devereaux PJ, Guyatt GH, Cook DJ, Swiotkowski MF, Sprague S, et al. An observational study of orthopedic abstracts and subsequent full-text publications. J Bone Joint Surg Am 2002; 84-A(4): 615-21.

6. Castillo J, García-Guasch R, Cifuentes I. Fate of abstracts from the Paris 1995 European Society of Anesthesiologists meeting. Eur J Anesthesiol 2002; 19(12): 888-93.

7. Hashkes P, Uziel Y. The publication rate of abstracts from the $4^{\text {th }}$ Park City Pediatric Rheumatology meeting in peer-reviewed journals: What factors influenced publication?. J Rheumatol 2003; 30(3): 597-602.

8. Hopewell S. Assessing the impact of abstracts from the Thoracic Society of Australia and New Zealand in Co- 
chrane reviews. Respirology 2003; 8: 509-12.

9. Krzyzanowska MK, Pintilie M, Tannock IF. Factors associated with failure to publish large randomized trials presented at an Oncology meeting. JAMA 2003; 290: 495-501

10. Scherer RW, Langenberg P, von Elm E. Full publication of results initially presented in abstracts. Cochrane Database Syst Rev. 2007, Apr 18(2): MR 000005.

11. Montané E, Vidal X. Fate of the abstracts presented at three Spanish clinical pharmacology congresses and reasons for unpublished research. Eur J Clin Pharmacol
2007; 63: 103-11

12. Uysal S, Tuglu B, Onvural B. Fate of abstracts presented at the 2002 IFCC Meeting. Clin Chem Lab Med 2008; 46(11): 1562-7.

13. Snedeker KG, Totton SC, Sargeant JM. Analysis of trends in the full publication of papers from conference abstracts involving pre-harvest or abbatoir-level interventions against foodborne pathogens. Preventive Veterinary Medicine 2010; 95 (1-2): 1-9.

14. Reyes H. Las referencias en artículos publicados en revistas biomédicas. Rev Med Chile 2001; 129: 343-5. 